\title{
Dielectric relaxations in ribose and deoxyribose supercooled water solutions
}

\author{
S. E. Pagnotta, ${ }^{1, a)}$ S. Cerveny, ${ }^{1}$ A. Alegría, ${ }^{1,2}$ and J. Colmenero ${ }^{1,2,3}$ \\ ${ }^{1}$ Centro de Fisica de Materiales (CSIC-UPV/EHU)-Materials Physics Center MPC, Universidad del Pais \\ Vasco (UPV/EHU), Apartado 1072, 20018 San Sebastian, Spain \\ ${ }^{2}$ Departamento de Fisica de Materiales, Facultad de Quimica, Universidad del Pais Vasco (UPV/EHU), \\ Apartado 1072, 20018 San Sebastian, Spain \\ ${ }^{3}$ Donostia International Physics Center, Paseo Manuel de Lardizabal 4, 20018 San Sebastian, Spain
}

(Received 27 May 2009; accepted 20 July 2009; published online 25 August 2009)

\begin{abstract}
The relaxation dynamic of ribose and deoxyribose water solutions at different concentrations has been studied by broadband dielectric spectroscopy and differential scanning calorimetry in the temperature range of 150-250 K. Two relaxation processes are observed for all the hydration levels; the slower (process I) is related to the relaxation of the whole solution whereas the faster one (process II) is associated with the reorientation of water molecules in the mixture. As for other polymeric water solutions, dielectric data for process II indicate the existence of a critical water concentration above which water mobility is less restricted. According to these results, attenuated total reflectance Fourier transform infrared spectroscopy measurements of the same sugar solutions showed an increment in the intensity of the $\mathrm{OH}$ stretching sub-band close to $3200 \mathrm{~cm}^{-1}$ as water content increases. (C) 2009 American Institute of Physics. [DOI: 10.1063/1.3202215]
\end{abstract}

\section{INTRODUCTION}

Ribose and 2'-deoxyribose are probably the most widespread monosaccharides in nature. They can be extensively found in ribonucleic acid (RNA) and 2'-deoxyribonucleic acid (DNA), respectively, where they form, together with a nitrogenous base and a phosphate group, a peculiar buildingblock structure called nucleotide. In particular, ribose is present in the nucleotide adenosine- $5^{\prime}$-triphosphate, a molecule that is known to be the major energy carrier of the cell and that regulates many biochemical pathways. ${ }^{1}$ In water solutions ribose and $2^{\prime}$-deoxyribose can be found both in their furanose and pyranose form, being the last one the most abundant (about $80 \%$ of the total ${ }^{2,3}$ ). As shown in Fig. 1, they posses a quite similar structure, i.e., a five (furanose) or sixmember (pyranose) ring composed of four or five carbon atoms and one oxygen. The only difference is the replacement, in the 2'-deoxyribose, of the hydroxyl group at the two position with hydrogen, leading to the net loss of an oxygen atom. These apparently small difference (and the presence in DNA molecules of the nitrogenous base thymine instead of uracil), entirely accounts for the differences between RNA and DNA. ${ }^{1}$ It is well known, for instance, that even if RNA could exist in a double stranded form, it is prevented from forming the double helix of B-DNA because of the steric interference by the $2^{\prime}$-hydroxyl group of its ribose units. ${ }^{4,5}$ Moreover, the same hydroxyl group, makes RNA more sensitive to hydrolysis of the phosphate diester backbone than DNA. ${ }^{1}$

In general, the study of the dynamical behavior of sugars (mono- and polysaccharides) posses an intrinsic interest, as they belong to a family of compounds able to undergo a

${ }^{\text {a)} E l e c t r o n i c ~ m a i l: ~ s c k p a g n s @ e h u . e s . ~}$ glass transition both in the anhydrous form and in solution with water. This phenomenon, in spite of the huge amount of work that has been devoted to the understanding of its occurrence in supercooled systems, still presents many unsolved aspects that need to be clarified (see Ref. 6 for a review). Moreover, the ability to vitrify an aqueous solution could be critically important in many biological situations in which biopreservation takes a role, and it is therefore the subject of an intense research. ${ }^{7-11}$ In this context, $2^{\prime}$-deoxyribose and ribose hold a major role because a complete characterization of the relaxation spectra of their water solutions could represent a first step toward a more ambitious task: the study of the dynamical behavior of complex macromolecules, such as DNA and RNA, in solution.

Broadband dielectric spectroscopy (BDS) is a well suited technique for the investigation of the molecular dynamics of glass forming water solutions, because of its sensitivity to the high dipolar moment of water molecules. Indeed, several studies applying BDS to different water-sugar<smiles>O[C@H]1CO[C@@H](O)C[C@H]1O</smiles>

Deoxyribopyranose<smiles>O[C@H]1[C@@H](O)[C@@H](O)[C@@H](O)[C@@H]1O</smiles>

Ribopyranose<smiles>OCC1O[C@@H](O)C[C@H]1O</smiles>

Deoxyribofuranose
FIG. 1. Pyranose and furanose form of $2^{\prime}$-deoxyribose and ribose. 
systems detected the presence of at least two dynamical phenomenon: a slower one, attributed to the collective movements of the system and a faster one, due to water molecules. $^{12-19}$ The relaxation time of the slower process usually follows a Vogel-Fulcher-Tammann ${ }^{20}$ (VFT) temperature dependence, typical of glass forming systems where the dynamics becomes frozen approaching low temperatures and quite similar to the well known $\alpha$ relaxation observed in supercooled systems above the glass transition temperature range. On the other hand the fast process, often associated with the reorientation of water dipoles in the solution, shows a temperature dependence that is Arrhenius-like below the glass transition of the system, indicating that in the low temperature range water motion could be in some way restricted by the glassy matrix. In this framework, while the relaxation spectra of 2 -deoxyribose and ribose anhydrous form have been recently published, ${ }^{17}$ a complete characterization of the two sugars at different hydration level is still missing.

In the present work BDS was used to study the dynamics of $2^{\prime}$-deoxyribose and ribose water solutions to gain a deeper insight into the nature of the relaxation processes of the sugar component of DNA and RNA. Measurements of Fourier transform infrared spectroscopy (FTIR) in attenuated total reflectance (ATR) geometry were also performed, representing an excellent opportunity for the study of hydrogen bonding. Indeed, the analysis of the intramolecular $\mathrm{OH}$ stretching mode is the most reliable method for probing, indirectly, the intramolecular network of water, since this contribution is sensitive to the level of interactions of the molecule with the surroundings. As in other glass forming water containing systems the two dielectric processes previously described were clearly identified and their temperature and hydration dependence investigated. In spite of the strong similarity between the two sugar molecules, $2^{\prime}$-deoxyribose possess an overall faster dynamics compared to ribose, presumably due to the possibility for the ribose molecule to form an extra hydrogen bond involving the hydroxyl group at the two position. Moreover, dielectric and infrared data for process II clearly indicate the existence of a critical water concentration above which water mobility is less restricted.

\section{EXPERIMENTAL}

D-ribose $\left(\mathrm{C}_{5} \mathrm{H}_{10} \mathrm{O}_{5}\right)$ and 2-deoxy-D-ribose $\left(\mathrm{C}_{5} \mathrm{H}_{10} \mathrm{O}_{5}\right)$ were purchased from Aldrich Chemical Co. and used without any further purification. Molecular weights for D-ribose and 2-deoxy-D-ribose are 150.13 and $134.13 \mathrm{~g} / \mathrm{mol}$, respectively. The corresponding chemical structures are shown in Fig. 1.

A differential scanning calorimeter (DSC) TA Instrument Q2000 was used in standard mode to monitor the crystallization of the samples and to measure the glass transition temperature. In particular, all the samples were sealed in hermetic aluminum pans and a cooling-heating cycle between $T_{g}-50 \mathrm{~K}$ and $T_{g}+50 \mathrm{~K}$, at a rate of $10 \mathrm{~K} / \mathrm{min}$, was performed using helium as transfer gas with a flow rate of 25 $\mathrm{ml} / \mathrm{min}$; the annealing time between cooling and heating runs was $2 \mathrm{~min}$. From the heat flow/temperature curves, $T_{g}$ values were calculated as the onset point.

A broadband high-resolution dielectric spectrometer, No-

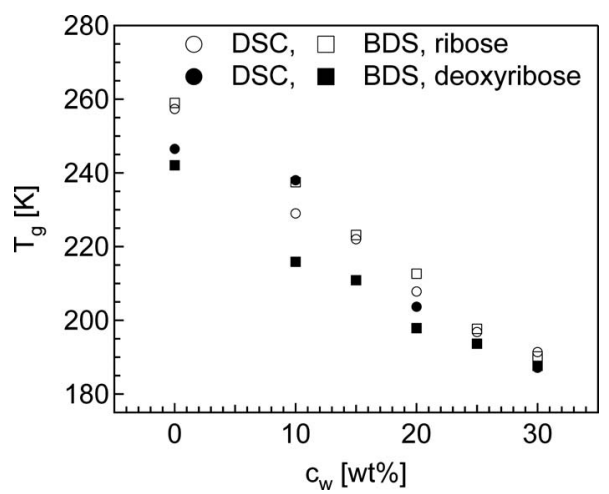

FIG. 2. Calorimetric and dielectric glass transition temperatures for ribose and deoxyribose samples at different water content, expressed in percentage as grams of water over grams of solution. Open symbols represents ribose solutions, while filled symbols represents deoxyribose solutions (circles refers to calorimetric glass transition $T_{g}$ and squares to dielectric $T_{D, 100 \mathrm{~s}}$ ).

vocontrol Alpha-N, was used to measure the complex dielectric function $\epsilon^{*}(\omega)=\epsilon^{\prime}(\omega)-\imath \epsilon^{\prime \prime}(\omega)(\omega=2 \pi f)$ in the frequency range of $10^{-2}-10^{7} \mathrm{~Hz}$; an Agilent rf impedance analyzer 4192B was used for the frequency range of $10^{6}-10^{9} \mathrm{~Hz}$. Parallel gold-plated electrodes were used, with a diameter of 20 and $10 \mathrm{~mm}$ for low and high frequency measurements, respectively. The sample thickness was typically $0.1 \mathrm{~mm}$. The isothermal frequency scans were performed every $5^{\circ}$ over the temperature range of $150-200 \mathrm{~K}$, and every $3^{\circ}$ from $203 \mathrm{~K}$ up to crystallization. The sample temperature was controlled with a stability better than $0.1 \mathrm{~K}$.

Anhydrous samples were prepared placing crystalline monosaccharides between two parallel gold-plated electrodes. The crystals were melted directly over them by keeping the temperature around their melting points, up to turn the samples transparent and care was taken to avoid thermal degradation. Then the samples were quenched putting them in liquid nitrogen to avoid any crystallization during cooling. Aqueous solutions were prepared dissolving the sugar with ultra pure water (Aldrich Chemical Co.) to a final water concentration ranging from $10 \%$ to $30 \%(\mathrm{w} / \mathrm{w})$; then they were placed in a liquid sample cell and again quenched in liquid nitrogen.

FTIR spectroscopy was carried out by means of a JASCO 6500 spectrometer using the ATR at room temperature in the range of $4500-700 \mathrm{~cm}^{-1}$. Each spectrum was collected with a resolution of $4 \mathrm{~cm}^{-1}$ and it is an average of 100 repetitive scans. The spectra were corrected for base line and penetration depth by using the software SPECTRA ANALYSIS from Jasco and no smoothing of the data was done. For each sample, the $\mathrm{OH}$ band was fitted using two or three Voigt functions according to similar previous studies. ${ }^{21-23}$

\section{RESULTS AND DISCUSSION}

A first characterization of the samples was made by means of DSC, in order to obtain the calorimetric glass transition temperatures $\left(T_{g}\right)$ as a function of water content. The maximum water content in the water-sugar solutions was kept under $30 \%$, to avoid any possible crystallization of the sample under the experimental conditions. As it is shown in Fig. 2 ribose posses a higher $T_{g}$ compared to that of deox- 

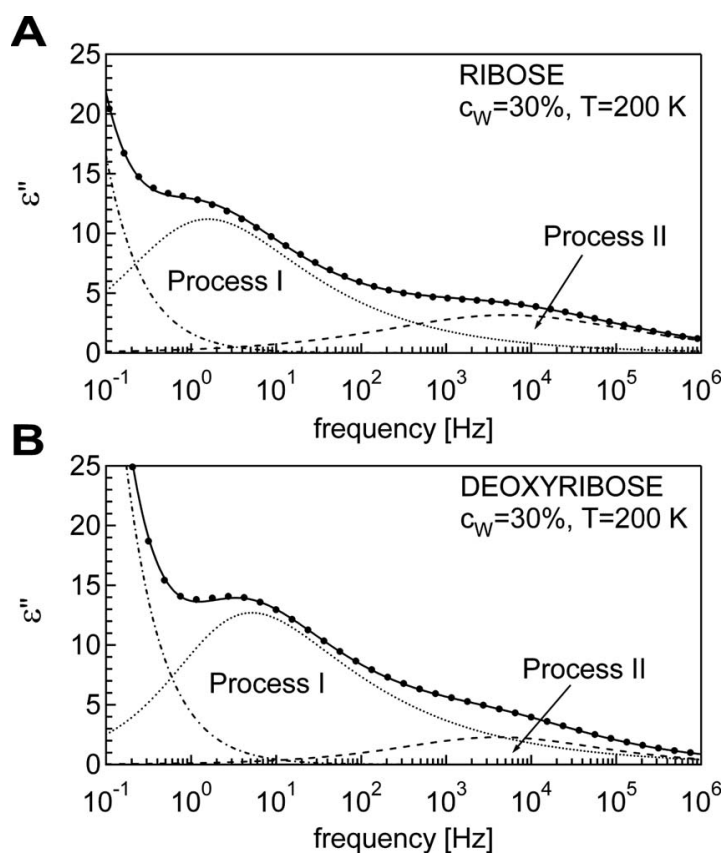

FIG. 3. Loss component $\epsilon^{\prime \prime}$ of the complex dielectric permittivity $\epsilon^{*}(f)$ of ribose (a) and deoxyribose (b) at a water content $c_{W}=30 \%$. Measurements are reported every $5^{\circ}$ over the temperature range of $155-200 \mathrm{~K}$, and every $3^{\circ}$ from 203 up to $233 \mathrm{~K}$.

yribose, at almost all the hydration level investigated in the present work. In the anhydrous sample this difference has been attributed to possible presence, in ribose, of an extra hydrogen bond at the $\mathrm{OH}$ group bound to the carbon 2, being this group missing, and substituted by a hydrogen, in deoxyribose (Fig. 1). ${ }^{17}$ As shown by the present data, this effect clearly tends to disappear with increasing water content, when the dynamics of the system starts to be more and more influenced by the dynamics of the solvent. Figure 2 reports, for comparison, also the value of the dielectric glass transition temperature, $T_{g, 100 \mathrm{~s}}$, calculated as the temperature at which the low frequency relaxation time reaches $100 \mathrm{~s}$ (see below).

Once assessed the calorimetric behavior, dielectric measurements were performed. Typical dielectric spectra for both ribose and deoxyribose solutions are depicted in Fig. 3 in the temperature range of $155-233 \mathrm{~K}$. The two sugars show a qualitative behavior quite similar to that of other small organic glass formers (see Ref. 18, and references therein), with the presence of two relaxation processes with characteristic temperature dependences. In particular, below the $T_{g}$ the isotherms present a wide relaxation (that we will denote, following the nomenclature in Ref. 18, as process II), while near $T_{g}$, isotherms also show a second relaxation (process I) in the low frequency range followed by process II at higher frequencies. It is important to mention here that even the dry samples presented two processes, at low and high frequencies. While the nature of the low frequency process is the same both in dry and hydrated samples, namely, a cooperative motion that in presence of water is plasticized and becomes faster, the nature of the high frequency process is quite different. In Ref. 17 the authors show that, as in other monosaccharides with comparable relaxation times, in the dry ribose and deoxyribose samples a fast secondary relaxation $(\gamma)$, of intramolecular origin, is present. This relaxation is tentatively ascribed to hydroxyl groups attached to the sugar ring. However, when we consider water solutions of ribose and deoxyribose, we have a new relaxation process superimposed to the $\gamma$ process of anhydrous samples, namely that one due to water dipole reorientation. As shown below, the activation energy of the two processes is quite different (higher for water dipole relaxation). Moreover, due to the greater dielectric strength of water process, at all hydration level investigated in the present work, the $\gamma$ process became almost undetectable, and was not considered further on in the fitting procedures.

To analyze the frequency dependence of the complex permittivities, we used the Havriliak-Negami (HN) equation ${ }^{24}$ plus a power law term to take into account the dc conductivity contribution. The equation finally reads

$$
\epsilon^{*}=\epsilon_{\infty}+\frac{\Delta \epsilon}{\left[1+\left(\iota \omega \tau_{\mathrm{HN}}\right)^{\alpha}\right]^{\gamma}}-\imath \frac{\sigma_{0}}{\epsilon_{0} \omega},
$$

where $\Delta \epsilon=\epsilon_{s}-\epsilon_{\infty}, \epsilon_{\infty}$, and $\epsilon_{s}$ being the unrelaxed and relaxed values of the dielectric constant, $\tau_{\mathrm{HN}}$ is the relaxation time, $\omega$ is the angular frequency and $\alpha$ and $\gamma$ are shape parameters $(\alpha>0, \alpha \gamma \leq 1)$. In particular, while for both ribose and deoxyribose solutions process I was well described by HN equation, process II was often fitted using a particular version of Eq. (1), in which the shape parameter $\gamma$ was set equal to one, obtaining in this way the symmetrical function called Cole-Cole, ${ }^{25}$ widely used to describe secondary relaxations in glassy materials. In order to obtain model-independent experimental results, the main relaxation times corresponding to processes I and II were then simply calculated from the loss maxima corresponding to the respective fitting curves, at constant temperature, as $\tau=1 / \omega_{\max }$. Figure 4 shows a typical example of such fitting procedures for ribose and deoxyribose samples at $30 \%$ water content and $T$ $=200 \mathrm{~K}$, with the two processes clearly resolved. In the following a detailed discussion of the different processes is reported.

\section{A. Process II}

The nature of process II has been recently discussed in several papers ${ }^{12,16,18,26-31}$ and attributed to the dynamics of the water molecules in the solution. In agreement with those works, we found that the dielectric strength of such process increases more rapidly at high water content [Fig. 5(a)], indicating an increase in the water mobility and a possible appearance of water domains. ${ }^{18,29}$ These findings are supported by a previous work ${ }^{32}$ investigating on water dynamics in a water-glucose solution. In that system, the authors showed how the rotational dynamics of water molecules localized within the glucose first hydration shell, where waterglucose interactions dominate, decrease by factors of 2-3 with respect to bulk water. Even if in the present study we are never dealing with bulk water, we can however still argue that in the samples with low water content the water dipole reorientation could be restricted by the binding with the sugar itself, while, on the other hand, at high water concen- 

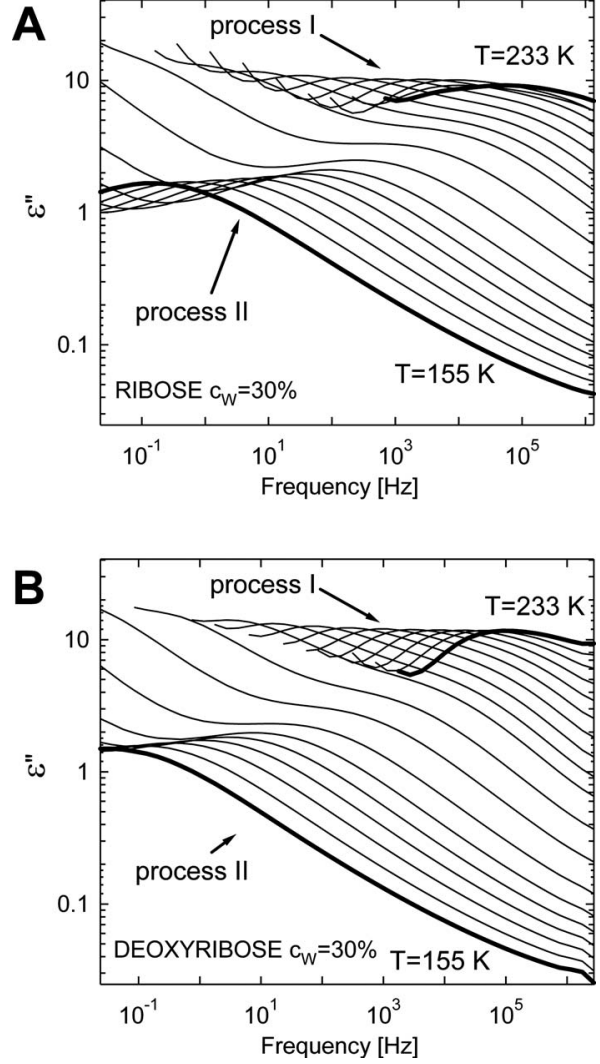

FIG. 4. Imaginary part of the complex permittivity for a ribose (a) and a deoxyribose (b) sample, at $T=200 \mathrm{~K}$ and $c_{W}=30 \%$. The solid line through the data points represents the overall least square fit. Dashed, dotted, and dotted-dashed lines represent process I, process II, and conductivity, respectively (see text).

tration, the number of water molecules surrounded by other water molecules increase, together with their ability to reorientate. To further support these considerations, we can observe, as reported in a previous paper, ${ }^{33}$ that the hydration of sugar molecules seems to be not simply related to the total number of $\mathrm{OH}$ groups or oxygen atoms present on the sugar itself. Indeed, the hydration properties may depend on the different conformations adopted by the monosaccharides in aqueous solutions, and in particular on a restricted number of $\mathrm{OH}$ groups: the equatorial ones. Uedaria and Uedaria $^{3}$ showed that in ribose and deoxyribose the mean number of equatorial $\mathrm{OH}$ groups is roughly equal to 2.14 and 1.37 , respectively. Interestingly, in good agreement with these values, in the present work the dielectric strength of process II

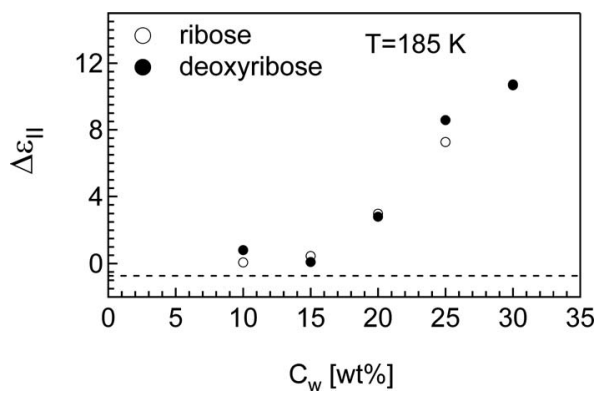

FIG. 5. Process II relaxation strength as a function of water content for ribose and deoxyribose samples at constant temperature. starts to increase more rapidly at a $c_{W}$ around 20\% (Fig. 5), a water concentration corresponding to 2.08 water molecules per ribose molecule and 1.86 water molecules per deoxyribose (see Table I). Thus, in both cases, it seems that $c_{W}$ $=20 \%$ could represent a critical water amount: under this hydration level water mainly interacts with the sugar molecules, while above it water-water correlations start to dominate the dielectric response.

These results found further confirmation when compared with ATR-FTIR measurements of the $\mathrm{OH}$ stretching band, performed on ribose and deoxyribose solutions in the 3000-3800 $\mathrm{cm}^{-1}$ range, at $298 \mathrm{~K}$ [Figs. 6(a) and 6(b)]. In general, as widely reported in literature, ${ }^{23,34}$ the $\mathrm{OH}$ stretching band of bulk water is decomposed into three main components that could be attributed to three types of water species. The first one, centered at about $3200 \mathrm{~cm}^{-1}$, corresponds to fully tetrahedrally coordinated hydrogen-bonded water molecules and represents the network coordination of bulk water. The second one, at higher frequencies (about $3600 \mathrm{~cm}^{-1}$ ), is ascribed to weakly hydrogen-bonded water molecules and it could represent small water aggregates or water having no contacts with other water molecules. Between these two extremes there are water molecules in an environment of partial hydrogen bond that contribute to the infrared spectra at intermediate frequencies (about $3400 \mathrm{~cm}^{-1}$ ). This last component could also reflect the interaction of water with a solute or a confining environment. ${ }^{23,35}$ Following this scheme we performed free fits of the $\mathrm{OH}$ stretching band data, as described in the experimental part, using two or three components depending on the sample water content. An example of such fitting procedure is depicted in Figs. 6(c) and 6(d), for ribose samples at $c_{W}=10 \%$ and $c_{W}=30 \%$. In particular, in both ribose and deoxyribose solutions at low water contents $\left(c_{W}<20 \%\right)$, only two components are necessary to correctly describe the data: one centered at about $3200 \mathrm{~cm}^{-1}$ and the other one close to $3400 \mathrm{~cm}^{-1}$. On the other hand, in ribose and deoxyribose samples at high water concentration, a third band appears at about $3500 \mathrm{~cm}^{-1}$. All these sub-bands did not vary significantly their frequency position in all the hydration range, with an average value of $3234 \pm 7,3404 \pm 16$, and $3557 \pm 9 \mathrm{~cm}^{-1}$ for ribose samples and $3235 \pm 8,3388 \pm 5$, and $3511 \pm 17 \mathrm{~cm}^{-1}$ for deoxyribose ones. However, when we looked at their intensities as a function of the water content the behavior was quite different (Fig. 7). Indeed, we observed that in both ribose and deoxyribose solutions, the sub-band at about $3400 \mathrm{~cm}^{-1}$ remains almost constant in the whole hydration range [Fig. 7(a)], while the sub-band at about $3200 \mathrm{~cm}^{-1}$ starts to increase more rapidly at a water content around 20\% [Fig. 7(b)]. From these results, and in analogy with the previously described classification used for ATR-FTIR spectra of bulk water, we could argue that the water molecules directly interacting with the sugar, and contributing in the intermediate range to the ATR-FTIR spectra (about $3400 \mathrm{~cm}^{-1}$ ), remains almost constant with increasing hydration levels. Conversely, the strongly hydrogen-bonded water molecules (with a full tetrahedrical coordination) increase with increasing hydration level, more rapidly above $c_{W}=20 \%$. Noteworthy, the relative abundance of these two 
TABLE I. Fitting parameters of processes I and II relaxation times. VFT function [Eq. (3)] was used for process I, while Arrhenius function [Eq. (2)] was used for process II.

\begin{tabular}{|c|c|c|c|c|c|c|c|c|}
\hline & \multirow[b]{2}{*}{$n_{\mathrm{H}_{2} \mathrm{O}}$} & \multirow[b]{2}{*}{$\begin{array}{c}c_{W} \\
(\%)\end{array}$} & \multicolumn{4}{|c|}{ VFT parameters of process I } & \multicolumn{2}{|c|}{ ARR parameters of process II } \\
\hline & & & $\log \tau_{\mathrm{VFT}}$ & $D_{T}$ & $\begin{array}{c}T_{0} \\
(\mathrm{~K})\end{array}$ & $m$ & $\log \tau_{\text {Arr }}$ & $\begin{array}{c}E_{a} \\
(\mathrm{eV})\end{array}$ \\
\hline \multirow[t]{6}{*}{ Ribose } & 0 & 0 & -14 & $8.3 \pm 0.4$ & $212.0 \pm 0.3$ & $89.9 \pm 0.1$ & $\cdots$ & $\cdots$ \\
\hline & 0.93 & 10 & -14 & $9.3 \pm 0.6$ & $190.0 \pm 0.3$ & $80.6 \pm 0.7$ & $-17.3 \pm 0.1$ & $0.53 \pm 0.01$ \\
\hline & 1.47 & 15 & -14 & $9.5 \pm 0.8$ & $177.8 \pm 0.4$ & $79.3 \pm 0.9$ & $-17.9 \pm 0.1$ & $0.57 \pm 0.01$ \\
\hline & 2.08 & 20 & -14 & $8.1 \pm 0.1$ & $174.1 \pm 0.1$ & $87.5 \pm 0.1$ & $-19.5 \pm 0.2$ & $0.65 \pm 0.01$ \\
\hline & 2.78 & 25 & -14 & $9.5 \pm 0.1$ & $157.1 \pm 0.1$ & $78.0 \pm 0.1$ & $-18.4 \pm 0.2$ & $0.59 \pm 0.01$ \\
\hline & 3.57 & 30 & -14 & $10.4 \pm 0.3$ & $148.2 \pm 0.9$ & $72.6 \pm 0.9$ & $-17.8 \pm 0.3$ & $0.55 \pm 0.01$ \\
\hline \multirow[t]{6}{*}{ Deoxyribose } & 0 & 0 & -14 & $10.5 \pm 0.1$ & $188.6 \pm 0.6$ & $72.8 \pm 0.9$ & $\ldots$ & $\ldots$ \\
\hline & 0.83 & 10 & -14 & $10.08 \pm 0.05$ & $165.6 \pm 0.2$ & $68.3 \pm 0.1$ & $-23.6 \pm 0.1$ & $0.75 \pm 0.05$ \\
\hline & 1.31 & 15 & -14 & $10.08 \pm 0.05$ & $165.6 \pm 0.2$ & $74.6 \pm 0.5$ & $-19.5 \pm 0.1$ & $0.63 \pm 0.01$ \\
\hline & 1.86 & 20 & -14 & $10.96 \pm 0.06$ & $152.6 \pm 0.2$ & $70.0 \pm 0.5$ & $-19.9 \pm 0.2$ & $0.68 \pm 0.01$ \\
\hline & 2.48 & 25 & -14 & $10.49 \pm 0.05$ & $150.8 \pm 0.2$ & $72.6 \pm 0.4$ & $-20.4 \pm 0.2$ & $0.69 \pm 0.01$ \\
\hline & 3.19 & 30 & -14 & $11.15 \pm 0.05$ & $144.1 \pm 0.2$ & $69.0 \pm 0.4$ & $-19.2 \pm 0.2$ & $0.62 \pm 0.01$ \\
\hline
\end{tabular}

water species reverts with increasing water content, being the sugar-interacting one predominant at low hydration level and the strongly hydrogen-bonded one more dominant at high hydration level. Finally, it is important to notice that the appearance in the ATR-FTIR spectra of a third component at about $3500 \mathrm{~cm}^{-1}$, when $c_{W} \geq 20 \%$, seems to indicate that with increasing hydration level the water behavior in ribose
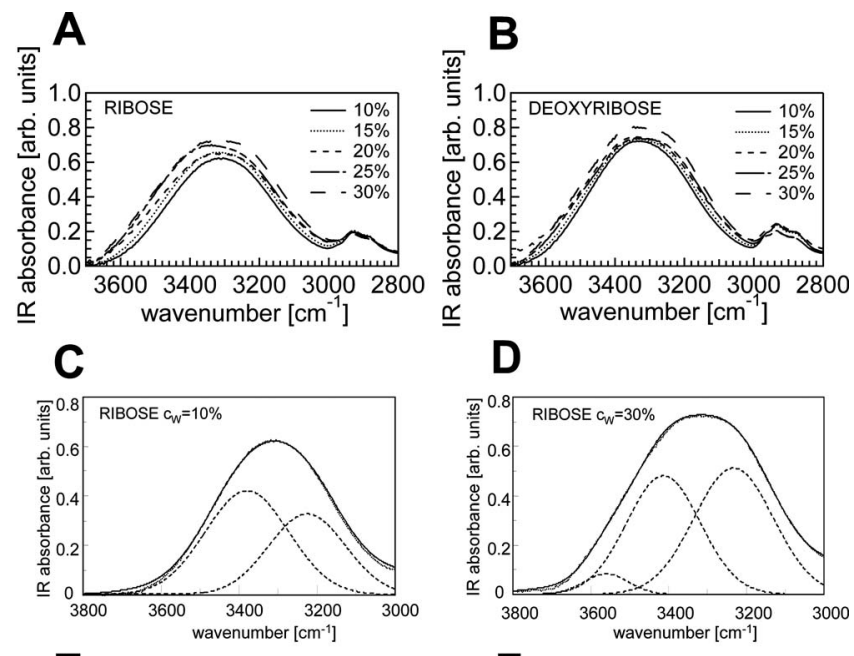

D

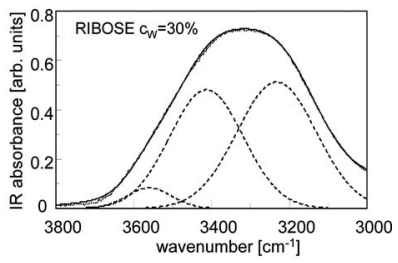

$\mathrm{E}$
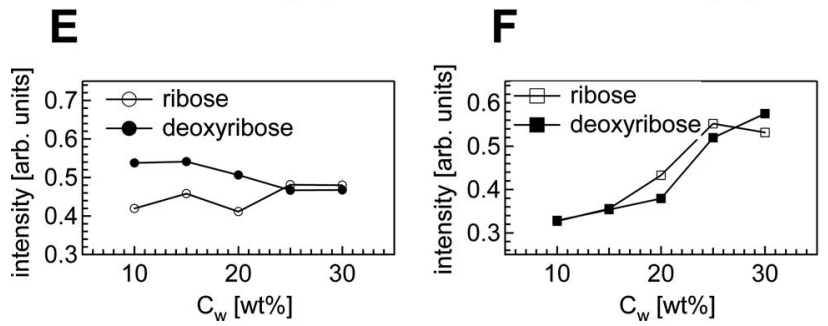

FIG. 6. ATR-FTIR raw data of ribose (a) and deoxyribose (b) solutions in the frequency range of $2800-3800 \mathrm{~cm}^{-1}$ at various water contents. The large $\mathrm{OH}$ stretching band is visible in the range of $3000-3800 \mathrm{~cm}^{-1}$, and a smaller contribution due to $\mathrm{CH}$ groups is also present in the 2800-3000 $\mathrm{cm}^{-1}$ range. Panels (c) and (d) show the least square fit of the $10 \%$ and $30 \%$ ribose solutions, obtained using two or three Voigt profiles, respectively (see text). Panels (e) and (f) report the intensities of the $\mathrm{OH}$ stretching sub-bands centered around 3400 and $3200 \mathrm{~cm}^{-1}$, respectively, for ribose and deoxyribose solutions as function of hydration level. and deoxyribose solutions tends to approach that one of bulk water, with the FTIR absorbance showing all the three characteristic components.

Putted together the BDS and ATR-FTIR data further validate the hypothesis that in both ribose and deoxyribose solutions, above a water content of about $20 \%$, the dynamical behavior of water could be dominated mainly by waterwater interactions. This conclusion is also supported by the temperature dependence analysis of process II relaxation times. In general, we can observe that below the glass transition, such temperature dependence is usually well described by the Arrhenius equation:

$$
\tau(T)=\tau_{\mathrm{Arr}} \exp \left[\frac{E_{a}}{R T}\right]
$$

where $\tau_{\text {Arr }}$ is a pre-exponential factor, $R$ is the gas constant, and $E_{a}$ is the apparent molar activation energy. As shown in Fig. 7, for all the samples investigated in the present work it was possible to fit process II data using Eq. (2), obtaining a set of parameters in good agreement with previous papers on other water solutions (see Table I). ${ }^{18,19,26}$

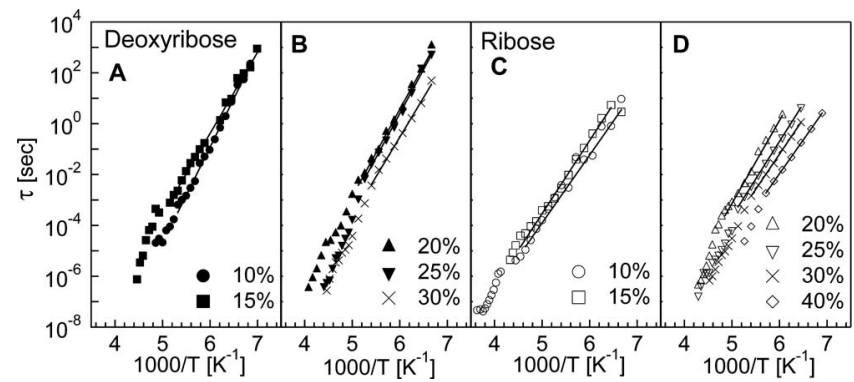

FIG. 7. Temperature dependence of the relaxation time $\tau(T)$ of process II. Panel (a) and (b): relaxation times for deoxyribose samples at different hydration levels. Solid lines through the data symbols represent an Arrhenius fit using Eq. (2). Parameters are listed in Table I. Panel (c) and (d): relaxation times for ribose samples at different hydration level. Solid lines through the data symbols represent an Arrhenius fit using Eq. (2). Parameters are listed in Table I. 
When considering the low temperature dynamics we found that the dielectric strength of process II is quite independent of water content, at low water concentration, and deoxyribose and ribose relaxation times do not vary significantly [Figs. 7(a) and 7(c)]. Contrary, at higher water content, when the dielectric strength starts to increase more rapidly [Fig. 5(a)], the dynamics of water dipole reorientation at a fixed temperature become faster as water concentration increases [Figs. 7(b) and 7(d)]. Noteworthy this effect is more pronounced for ribose solutions, apparently indicating that in these samples, water dynamics is still strongly influenced by the interaction with the sugar. Contrary, in deoxyribose solutions, the relaxation times are less dependent of water content.

As already reported in several papers on the dynamics of water in solution or hard confinement (see Ref. 18, and references therein), the relaxation times showed a crossover at about $T_{g}$, more evident at high water content and in ribose samples, from Arrhenius to apparently non-Arrhenius with increasing temperatures. This crossover could be alternatively due to different Arrhenius dependences at low and high temperatures. However, this last interpretation would lead to unrealistically low values of the pre-exponential factors in the Arrhenius equation.

Focusing again on the low temperature regime, the extremely low values of $\tau_{\text {Arr }}$ reported in Table I suggest the movement of more than one unit, since it does not fit the Starkweather's definition of a simple relaxation. ${ }^{36,37}$ This phenomenon could be analogously rationalized within the frame of transition state theory, supposing the existence of a distribution of activation entropy barriers, with a high mean activation entropy, mainly of a configurational origin. ${ }^{38} \mathrm{Be}-$ ing this effects already noticeable for low water content, water molecules could be not uniformly distributed, hydrating first the more favorable sites and giving rise to some kind of aggregate where they could move together.

Finally, as shown in Table I in the deoxyribose and ribose samples we can observe an overall high activation energy, almost independent of hydration level, and generally higher for deoxyribose samples than for ribose ones. Again this observation may reflect to some extent the more cooperative character shown by water molecules in the deoxyribose solution, with respect to that one in ribose solutions.

\section{B. Process I}

Differently from process II, the process I dynamics reported in Fig. 8 follows a VFT temperature dependence:

$$
\tau(T)=\tau_{\mathrm{VFT}} \exp \left[\frac{D_{T} T_{0}}{T-T_{0}}\right]
$$

where $\tau_{\mathrm{VFT}}$ is a pre-exponential factor, $D_{T}$ is a parameter related to fragility, ${ }^{39}$ and $T_{0}$ is the temperature at which the dynamics of the system diverge. Such behavior is generally considered to arise from the cooperative rearrangements of the sugar and water molecules. Noteworthy, the temperature values at which the relaxation times reach 100 s, i.e., the dielectric $T_{g, 100 \mathrm{~s}}$, show an overall good agreement with the corresponding calorimetric values for all the investigated
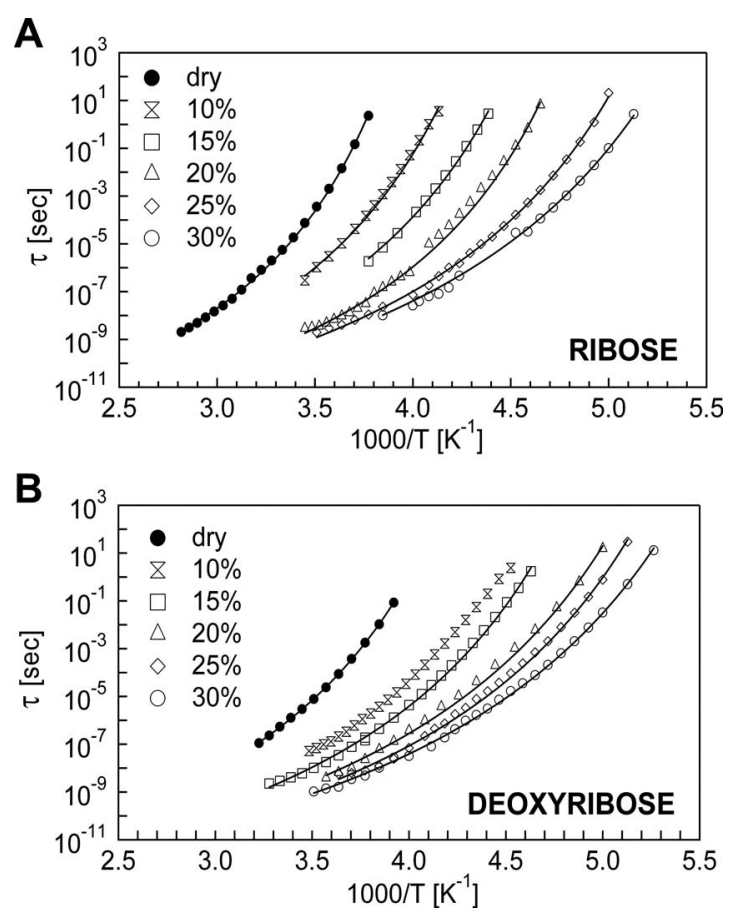

FIG. 8. Temperature dependence of the relaxation time $\tau(T)$ for process I. Panel (a) and (b): relaxation times for ribose and deoxyribose samples, respectively, at different hydration levels. Solid lines through the data symbols represent a VFT fit using Eq. (3). Parameters are listed in Table I.

samples (see Fig. 2), further supporting the idea of a global process strongly resembling the $\alpha$-relaxation observed in supercooled systems above the $T_{g}$. The addition of water to the anhydrous sugars always produces systems with faster dynamics, with deoxyribose solutions generally slightly faster than the ribose one. However this difference tends again to disappear with increasing dilution [as seen in Figs. 8(a) and 8(b)], probably due to the stronger contribution of water dynamics on the global process. Moreover, a close inspection of the VFT parameters reported in Table I (where $\tau_{\mathrm{VFT}}$ was fixed to $10^{-14}$, close to the reciprocal of typical vibrational frequencies) reveals that both ribose and deoxyribose solutions posses a moderately fragile character at all water contents, with generally higher values for the ribose system, as indicated by $D_{T}$ and the fragility parameter $m$ [defined as $m=d \log \tau /\left.d\left(T_{g, 100 \mathrm{~s}} / T\right)\right|_{T=T_{g, 100 \mathrm{~s}}} .^{39}$

\section{CONCLUSIONS}

Ribose and deoxyribose water solution in the range of 0\%-30\% of water content have been characterized by means of BDS, DSC, and ATR-FTIR. The two systems, both possessing a moderately fragile character, show an overall similar behavior, namely, the presence of two main relaxation process. The faster one (process II) is related to the orientational dynamics of the water molecules and shows an Arrhenius behavior below $T_{g}$, while the slower one (process I) arises from the cooperative rearrangement of the whole system and possesses a VFT dynamics. In spite of the strong similarity between the ribose and deoxyribose molecules (see Fig. 1), both BDS and DSC techniques are able to detect a difference in the dynamical properties of the two sugars in 
solution. The presence in the ribose of an extra $\mathrm{OH}$ group, substituted in deoxyribose by a simple hydrogen, lead to an overall higher $T_{g}$, and a slower dynamics of the system, an effect that is stronger in the anhydrous samples and tends to disappear with increasing amount of water.

The possibility for the ribose molecule to form an extra hydrogen bond involving the hydroxyl group at the 2 position, probably accounts for such differences. Indeed, in the anhydrous sugar the higher glass transition temperature could arise from the extra energy necessary to break this bond. Moreover, the addition of increasing amounts of water to the system clearly reduces the importance of this contribution, and the dynamical behavior of the solvent starts to be predominant. This last observation was also supported by the combined use of BDS and ATR-FTIR techniques, clearly pointing out a threshold behavior for the water dynamics in the sugar solutions. Indeed, in samples with a water concentration higher than about $20 \%$ water-water interactions start to be predominant driving the dynamic of the whole system.

\section{ACKNOWLEDGMENTS}

The authors gratefully acknowledge the support of the Spanish Ministry of Education, project CSD2006-00053, the European Union, project 502235-2 and SOFTCOMP program and the Basque Government, project IT-436-07.

${ }^{1}$ L. Stryer, Biochemistry, 4th ed. (Freeman, New York, 1999).

${ }^{2}$ S. J. Angyal and V. A. Picles, Aust. J. Chem. 25, 1695 (1972).

${ }^{3}$ H. Uedaira and H. Uedaira, J. Solution Chem. 14, 27 (1985).

${ }^{4}$ J. J. Butzow and G. L. Eichhorn, Nature (London) 254, 358 (1975)

${ }^{5}$ M. Komiyama, N. Takeda, and H. Shigekawa, Chem. Commun. (Cambridge) 1999,1443

${ }^{6}$ C. A. Angell, K. L. Ngai, G. B. McKenna, P. F. McMillan, and S. W. Martin, J. Appl. Phys. 88, 3113 (2000).

${ }^{7}$ J. H. Crowe, J. F. Carpenter, and L. M. Crowe, Annu. Rev. Physiol. 60, 73 (1998).

${ }^{8}$ J. Buitink and O. Leprince, Cryobiology 48, 215 (2004)

${ }^{9}$ J. L. Green and C. A. Angell, J. Phys. Chem. 93, 2880 (1989).

${ }^{10}$ L. Cordone, G. Cottone, and S. Giuffrida, J. Phys.: Condens. Matter 19, 205110 (2007)

${ }^{11}$ S. E. Pagnotta, M. A. Ricci, F. Bruni, S. E. McLain, and S. Magaz, Chem. Phys. 345, 159 (2008).

${ }^{12}$ H. Jansson, R. Bergman, and J. Swenson, J. Non-Cryst. Solids 351, 2858 (2005).

${ }^{13}$ H. P. Cummins, H. P. Zhang, J. Y. Oh, J. A. Seo, H. K. Kim, Y. H.
Hwang, Y. S. Yang, Y. S. Yu, and Y. Inn J. Non-Cryst. Solids 352, 4464 (2006).

${ }^{14}$ Y. H. Hwang, J. Seo, H. Kwon, and H. K. Kim, Rep. Inst. Fluid Science 19, 27 (2007).

${ }^{15}$ I. Ermolina, E. Polygalov, C. Bland, and G. Smith, J. Non-Cryst. Solids 353, 4485 (2007).

${ }^{16}$ N. Shinyashiki, S. Sudo, S. Yagihara, A. Spanoudaki, A. Kyritsis, and P. Pissis, J. Phys.: Condens. Matter 19, 205113 (2007).

${ }^{17}$ K. Kaminski, E. Kaminska, P. Wlodarczyk, M. Paluch, J. Ziolo, and K. L. Ngai, J. Phys.: Condens. Matter 20, 335104 (2008).

${ }^{18}$ S. Cerveny, A. Alegría, and J. Colmenero, Phys. Rev. E 77, 031803 (2008).

${ }^{19}$ N. Shinyashiki, M. Shinohara, Y. Iwata, T. Goto, M. Oyama, S. Suzuki, W. Yamamoto, S. Yagihara, T. Inoue, S. Oyaizu, S. Yamamoto, K. L. Ngai, and S. Capaccioli, J. Phys. Chem. B 112, 15470 (2008).

${ }^{20}$ H. Vogel, Phys. Z. 22, 645 (1921); G. S. Fulcher, J. Am. Chem. Soc. 8, 339 (1925); G. Tammann and W. Hesse, Z. Anorg. Allg. Chem. 156, 245 (1926).

${ }^{21}$ M. B. Temsamani, M. Maeck, I. El Hassani, and H. D. Hurwitz, J. Phys. Chem. B 102, 3335 (1998)

${ }^{22}$ J. B. Brubach, A. Mermet, A. Filabozzi, P. Colavita, A. Gerschel, D. Lairez, and P. Roy, J. Phys. Chem. B 105, 430 (2001).

${ }^{23}$ V. Crupi, F. Longo, D. Majolino, and V. Venuti, J. Chem. Phys. 123, 154702 (2005).

${ }^{24}$ S. Havriliak and S. Negami, Polymer 8, 161 (1967).

${ }^{25}$ R. H. Cole and K. S. Cole, J. Chem. Phys. 10, 98 (1942).

${ }^{26}$ S. Cerveny, A. Alegría, and J. Colmenero, J. Chem. Phys. 128, 044901 (2008).

${ }^{27}$ S. Sudo, S. Tsubotani, M. Shimomura, N. Shinyashiki, and S. Yagihara, J. Chem. Phys. 121, 7332 (2004).

${ }^{28}$ S. Cerveny, J. Colmenero, and A. Alegría, Macromolecules 38, 7056 (2005).

${ }^{29}$ S. Cerveny, A. Alegria, and J. Colmenero, J. Chem. Phys. 124, 194501 (2006).

${ }^{30}$ S. Cerveny, J. Colmenero, and A. Alegría, J. Non-Cryst. Solids 353, 4523 (2007).

${ }^{31}$ S. Capaccioli, K. L. Ngai, and N. Shinyashiki, J. Phys. Chem. B 111, 8197 (2007).

${ }^{32}$ M. Paolantoni, P. Sassi, A. Morresi, and S. Santini, J. Chem. Phys. 127, 024504 (2007).

${ }^{33}$ M. J. Tait, A. Suggett, F. Franks, S. Ablett, and P. A. Quickenden, J. Solution Chem. 1, 131 (1972).

${ }^{34}$ C. Boissiere, J. B. Brubach, A. Mermet, G. de Marzi, C. Bourgaux, E. Prouzet, and P. Roy, J. Phys. Chem. B 106, 1032 (2002).

${ }^{35}$ V. Crupi, F. Longo, D. Majolino, and V. Venuti, J. Phys.: Condens. Matter 18, 3563 (2006).

${ }^{36}$ H. W. Starkweather, Macromolecules 14, 1277 (1981).

${ }^{37}$ H. W. Starkweather, Polymer 32, 2443 (1991).

${ }^{38}$ A. Kudlik, C. Tschirwitz, T. Blochwicz, S. Benkhof, and E. Rossler, J. Non-Cryst. Solids 235-237, 406 (1998).

${ }^{39}$ R. Bohmer, K. L. Ngai, C. A. Angell, and D. J. Plazek, J. Chem. Phys. 99, 4201 (1993). 